\title{
Gamma Ray Pulsars: Observations
}

\author{
David J. Thompson \\ Laboratory for High Energy Astrophysics \\ NASA Goddard Space Flight Center \\ Greenbelt, Maryland 20771 USA
}

\begin{abstract}
High-energy gamma rays are a valuable tool for studying particle acceleration and radiation in the magnetospheres of energetic pulsars. The six or more pulsars seen by CGRO/EGRET show that: the light curves usually have double-peak structures (suggesting a broad cone of emission); gamma rays are frequently the dominant component of the radiated power; and all the spectra show evidence of a high-energy turnover. Unless a new pulsed component appears at higher energies, progress in gamma-ray pulsar studies will be greatest in the $1-20 \mathrm{GeV}$ range. Ground-based telescopes whose energy ranges extend downward toward $10 \mathrm{GeV}$ should make important measurements of the spectral cutoffs. The Gamma-ray Large Area Space Telescope (GLAST), now in planning for a launch in 2005, will provide a major advance in sensitivity, energy range, and sky coverage.
\end{abstract}

\section{INTRODUCTION}

Pulsars are particularly interesting astrophysical objects because we know so much about them, and much of that information is derived from their timing properties. Measurement of the period $\mathrm{P}$ and period derivative $\dot{P}$ first identified pulsars as compact objects. Under fairly general assumptions that pulsars are rapidly- rotating neutron stars with a strong dipole magnetic field, a whole range of physical parameters can be estimated from these timing parameters. Examples include the timing age, the spin-down energy loss, the surface magnetic field, and the open field line voltage [1].

\section{THE OBSERVED GAMMA-RAY PULSARS}

The telescopes on the Compton Gamma Ray Observatory identified a number of gamma-ray pulsars, some with very high confidence and others with less certainty. There are at least three answers to the question, "How many gamma-ray pulsars are there?"

The first answer is that there are seven or more gamma-ray pulsars. Figure 1 shows their light curves in four energy bands: roughly $0.5-2 \mathrm{KeV}, 2-100 \mathrm{keV}, 100$ 
$\mathrm{keV}-10 \mathrm{MeV}$, and above $100 \mathrm{MeV}$. All seven of these are positive detections in the gamma-ray band above $100 \mathrm{KeV}$. The weakest (PSR B1951+32) has a statistical probability of occurring by chance of $\sim 10^{-9}$.

Some important features of these pulsar light curves are:

- They are not the same at all wavelengths. Some combination of the geometry

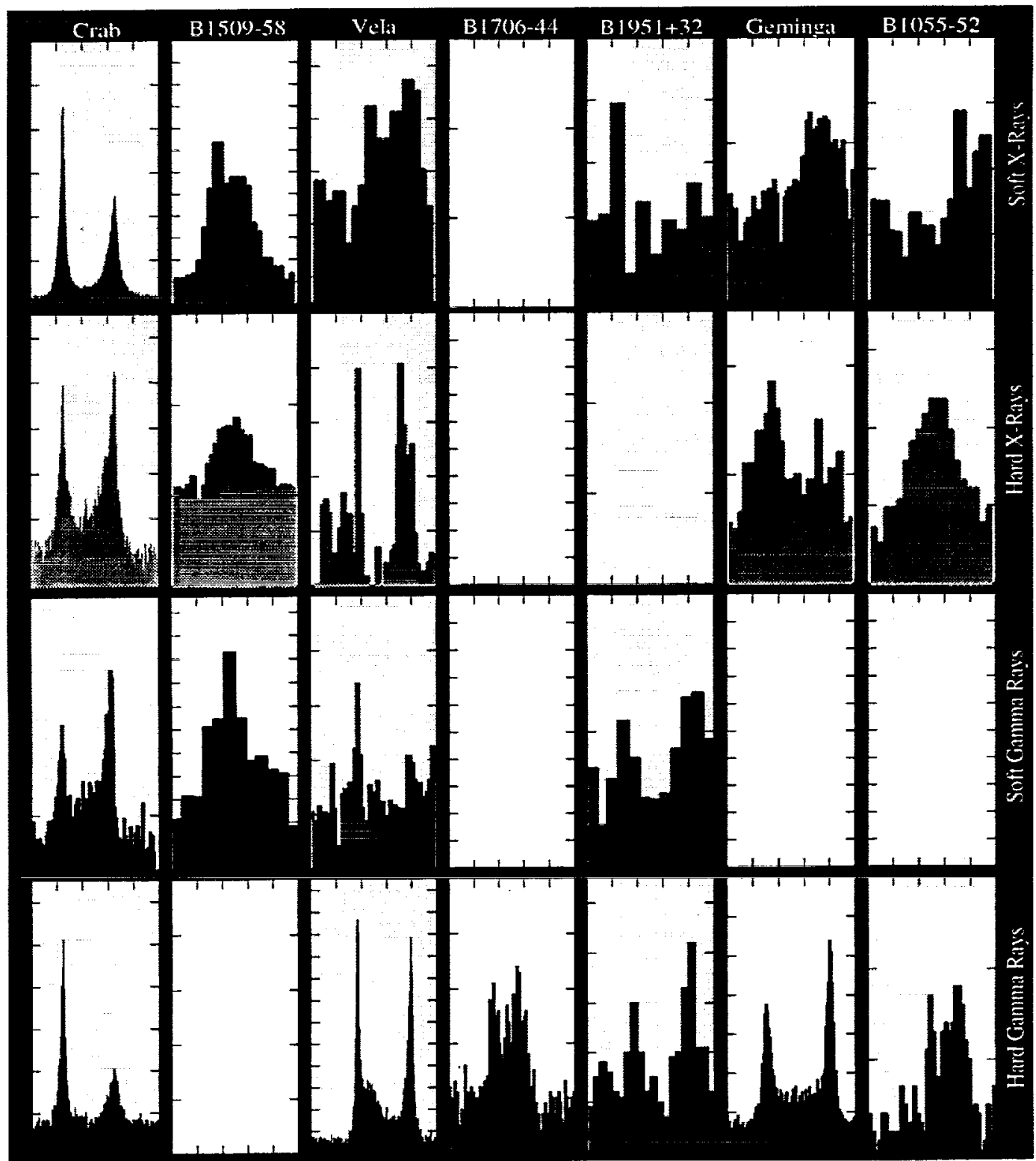

FIGURE 1. Light curves of seven gamma-ray pulsars in X-rays and gamma rays, from left to right in order of timing age. Each panel shows one full rotation of the neutron star. Ref. Crab: [2-4, this work]; PSR B1509-58: [5-7]; Vela: [8-11]; PSR B1706-44: [12]; PSR B1951+32: [13-15]; Geminga: [16, this work]; PSR B1055-52: [17]. 
and the emission mechanism is energy-dependent. In soft X-rays, for example, the emission in same cases seems to be thermal, probably from the surface of the neutron star; clearly this is not the case for gamma radiation.

- Not all seven are seen at the highest energies. PSR B1509-58 is seen only up to $10 \mathrm{MeV}$, by COMPTEL [7], and not above $100 \mathrm{MeV}$ by EGRET. For this reason, the answer to the question, "How many high-energy gamma ray pulsars are seen?" is, "At least six."

- The six seen by EGRET all have a common feature - they show a double peak in their light curves. Because these high-energy gamma rays are associated with energetic particles, it seems likely that the particle acceleration and interactions are taking place along a large hollow cone or surface.

In addition to the six high-confidence pulsar detections above $100 \mathrm{MeV}$, three additional radio pulsars may have been seen by EGRET. Figure 2 shows their light curves (without the zero suppression used in some of the original references). These three all have statistical probabilities in the $10^{-4}$ range, or about 5 orders of magnitude less convincing than the weakest of the seven on the previous figure. These

B1046-58

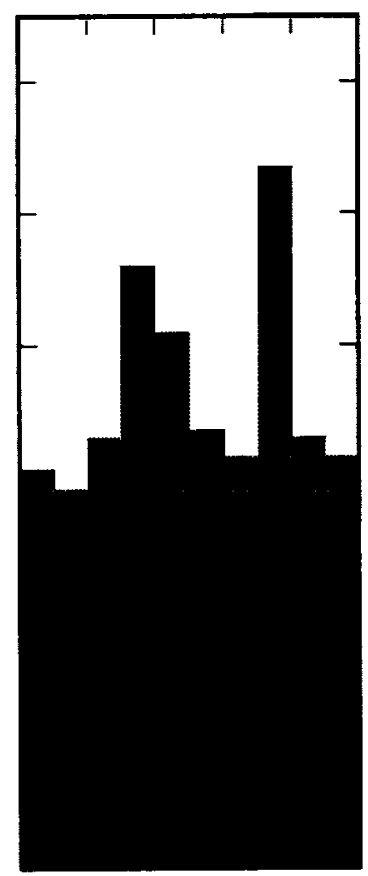

B0656+14

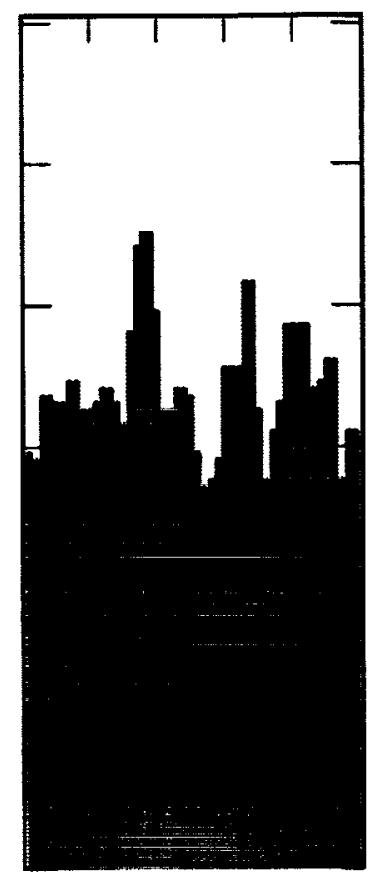

$\mathrm{J0218}+4232$

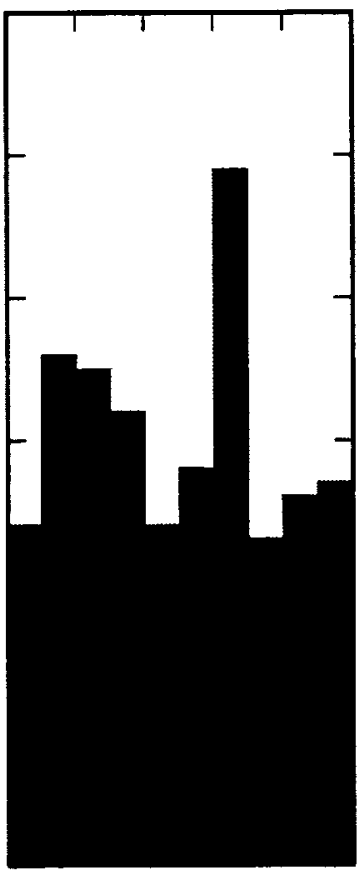

FIGURE 2. Light curves of three candidate gamma-ray pulsars. Each panel shows one full rotation of the neutron star. References: PSR B1046-58: [18]; PSR B0656+14: [19]; PSR J0218+4232 [20]. 
are good candidates, but they are not strong enough to be used as discriminators between models. These three do imply a third answer to the "how many" question - there could be as many as ten known gamma-ray pulsars.

This sample of pulsars can be compared to other pulsars in terms some of the derived physical parameters. Figure 3 is a distribution of pulsars as a function of their period and period derivative, with the gamma-ray pulsars shown as large dots.

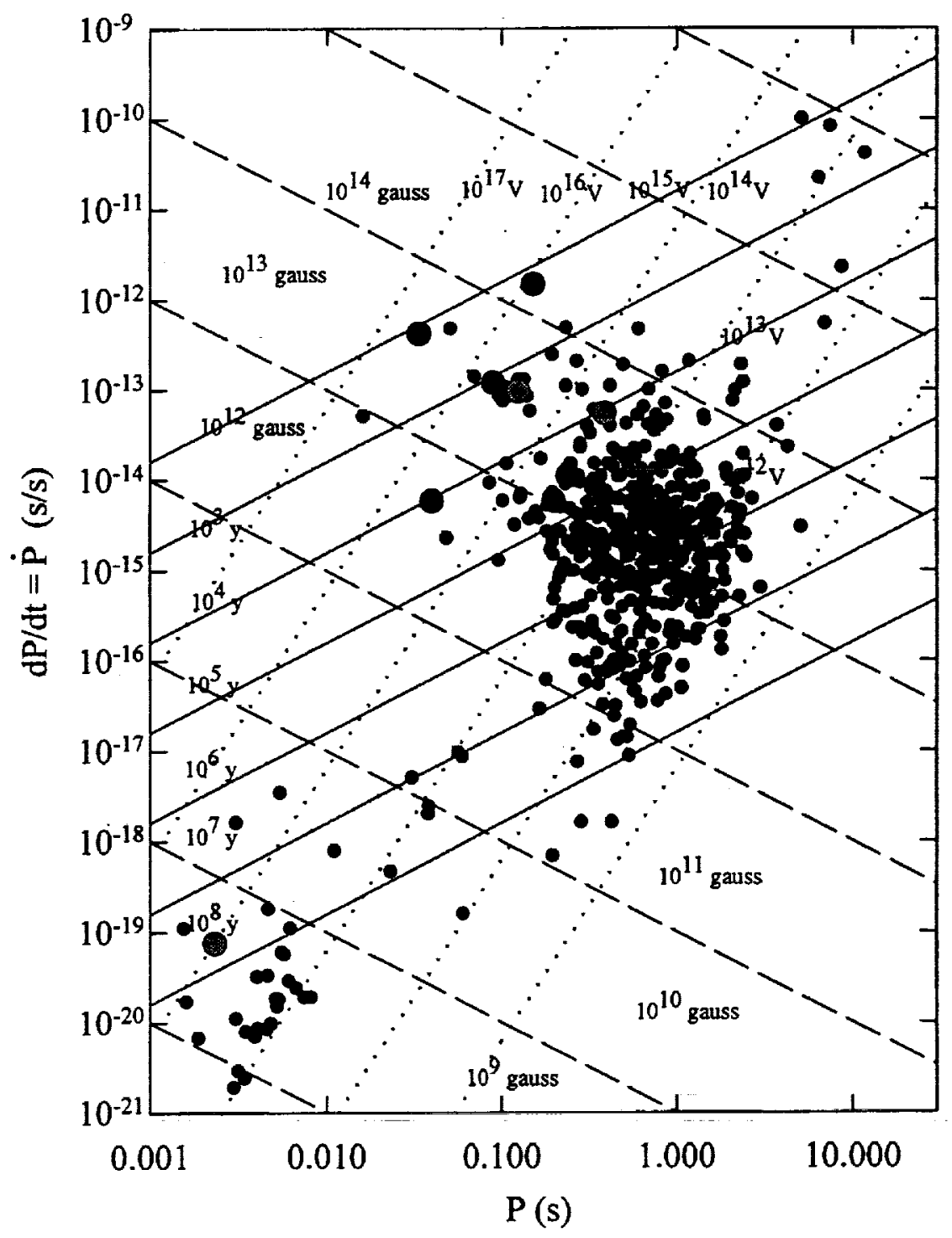

FIGURE 3. Period v. period derivative for a large sample of pulsars. Small dots: no gamma-ray emission. Large dark dots: seven high-confidence gamma-ray pulsars. Large light dots: three lower-confidence gamma-ray pulsars. Solid lines: timing age. Dotted line: open field line voltage. Dashed line: surface magnetic fields. 
Also shown are some of the derived physical parameters. The gamma-ray pulsars tend to be concentrated (with the exception of the one millisecond candidate) in a region with high magnetic field (but not magnetar-strength) - shown by the dashed lines, and relatively young ages - shown by the solid lines. All ten gamma-ray pulsars share a third characteristic, shown by the dotted line, of having the open field line voltage high compared to most pulsars. This is not surprising, since the particles are being accelerated electromagnetically.

The open field line voltage, which is also proportional to the polar cap (GoldreichJulian) current, has long been recognized as a significant parameter for gamma-ray pulsars [22]. Figure 5 illustrates that the pulsar high-energy luminosities, integrated above $1 \mathrm{eV}$, are approximately proportional to this parameter, shown by the solid line in the figure. An interesting question is what happens for lower voltages, where the high-energy luminosity converges with the total spin-down energy available, shown by the dashed line. This will be a question for future missions.

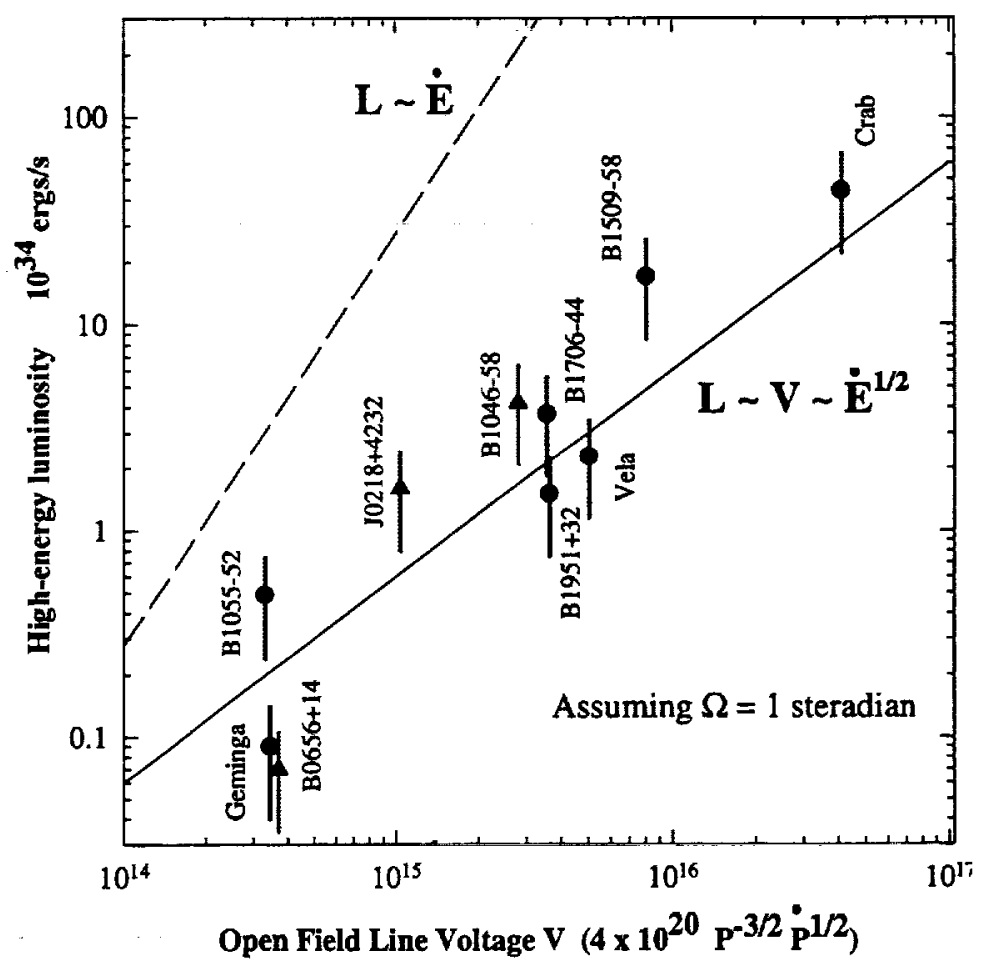

FIGURE 4. High-energy pulsar luminosity as a function of the open field line voltage. Circles: high-confidence pulsars; Triangles: lower-confidence pulsars. 


\section{PULSARS AT THE HIGHEST ENERGIES}

What happens to pulsar observations at the highest energies? No pulsars have been seen at $\mathrm{TeV}$ energies. The upper end of the EGRET range represents the highest energies for detections of pulsed emission. There is evidence of pulsed emission above $5 \mathrm{GeV}$ for all six of the pulsars definitely seen by EGRET. Figure 5 shows the light curves for the four with the best statistics (the other two are consistent with these), shown in the upper panels in the $100 \mathrm{MeV}$ range, and at the bottom for photons above $5 \mathrm{GeV}$. It is clear that something has changed in the multi-GeV light curves - each of the pulsars above $5 \mathrm{GeV}$ is dominated by one of the two pulses seen at lower energies. In fact, for all except PSR B1706-44, it is the trailing pulse that dominates. Because of EGRET's very low background, the second pulse is still barely visible. A detector with higher background would see only the single pulse. These observations suggest that this energy range is a critical

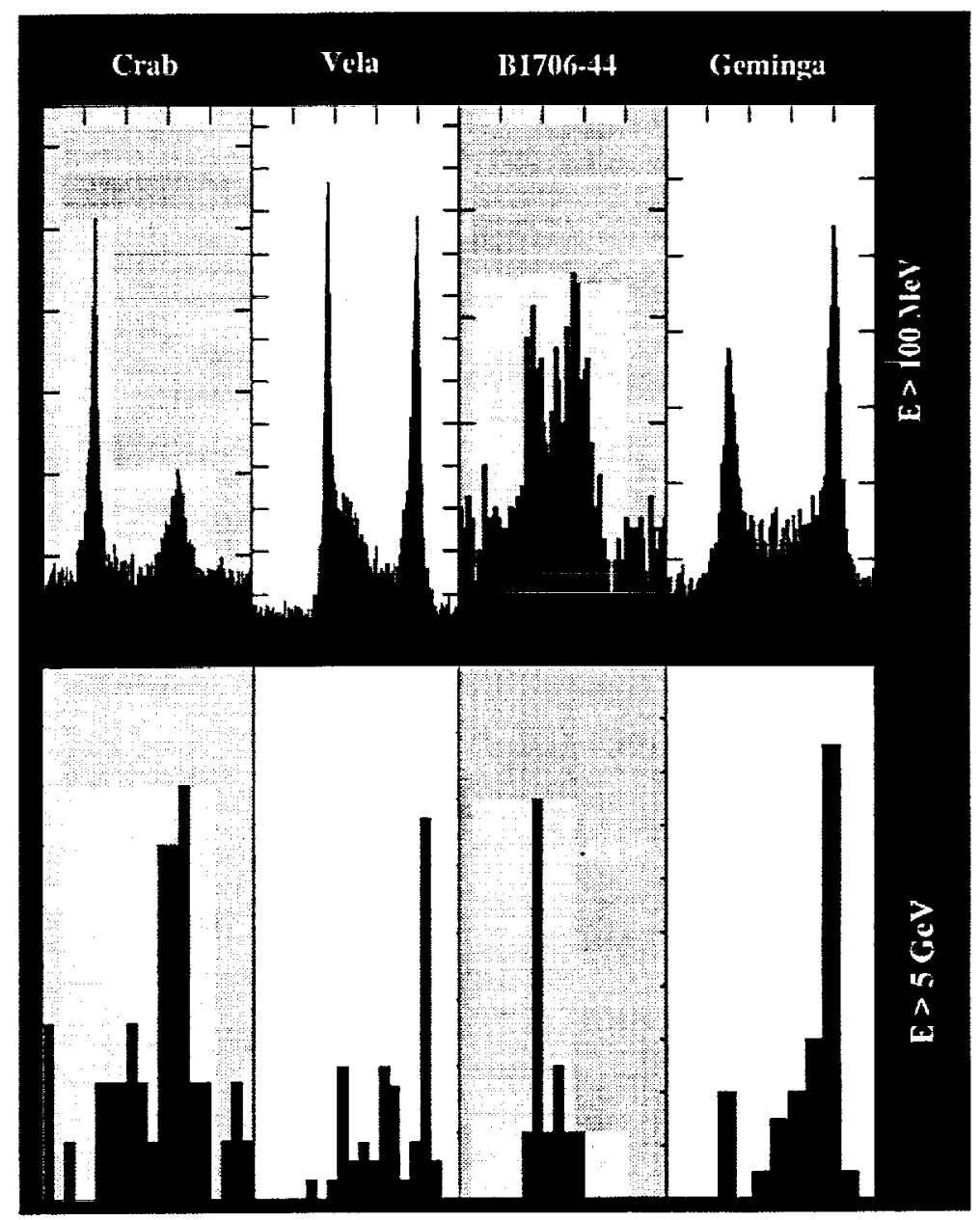

FIGURE 5. Light curves of four gamma-ray pulsars above $100 \mathrm{MeV}$ and above $5 \mathrm{GeV}$. Each panel shows one full rotation of the neutron star. 
one for gamma-ray production.

The importance of the $1-20 \mathrm{GeV}$ band is also visible in the energy spectra [17]. In all gamma-ray pulsars, the dominant power is seen in the hard $\mathrm{x}$-ray to hard gamma-ray band - between $100 \mathrm{keV}$ for the $\mathrm{Crab}$ and greater than $10 \mathrm{GeV}$ for PSR B1951+32. Also in all cases, there is a fall-off at higher energies. The upper limits from the ground-based detectors are typically an order of magnitude or more below the peak luminosity. The gap between about $10 \mathrm{GeV}$ (where EGRET runs out of photons) and the current generation of ground-based telescopes is very important. It is hard to predict from the limited EGRET data what is expected even at 100 $\mathrm{GeV}$. Figure 6 shows the spectrum of Vela, the brightest of the EGRET pulsars. This spectrum is shown in $\nu \mathrm{F} \nu$ or power per logarithmic energy interval format. The $10-30 \mathrm{GeV}$ point is based on only 4 photons, and the absolute calibration of EGRET at these energies is fairly uncertain. Also shown are the spectra expected in two popular gamma-ray pulsar models, the polar cap [23,24] and the outer gap $[25,26]$. The large error bars on the EGRET data make them consistent with both models. The extrapolation to higher energies is, however, dramatically different. There is also the possibility of a second, inverse-Compton component of the pulsed radiation at higher energies, expected in some outer gap models [25], although there is yet no observational evidence for that component. Searching for that second component will be an important goal of the next generation of very-high-energy gamma-ray telescopes.

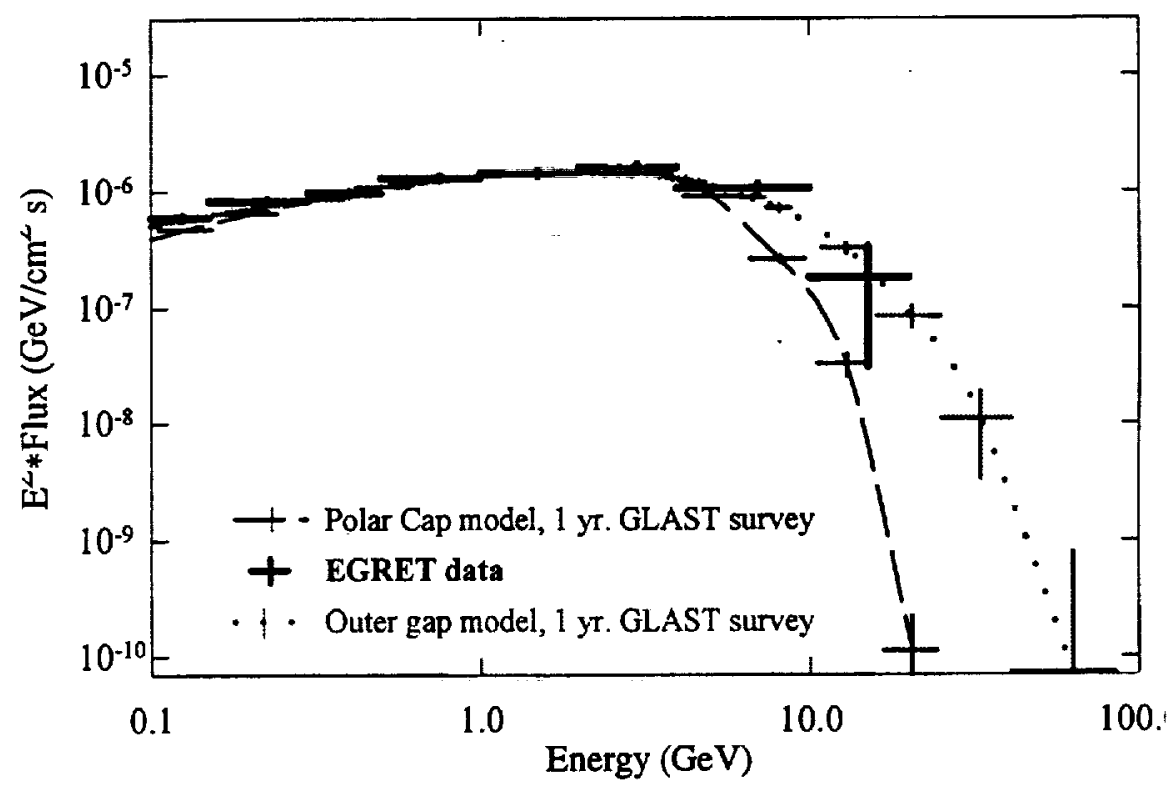

FIGURE 6. High-energy spectrum of the Vela pulsar. Heavy error bars: EGRET data [27]. Dotted line: Outer gap model [25,26]. Dashed line: Polar cap model [23,24]. Error bars shown on the models are those expected from the GLAST mission [28] in a one-year sky survey. 


\section{FUTURE PULSAR OBSERVATIONS AT HIGH ENERGIES}

The high luminosity in the GeV energy range and the drop-off above that part of the spectrum suggest that GLAST will be the next big step in gamma-ray pulsar observations. AGILE will certainly make a contribution, especially in confirming those candidate pulsars for which the EGRET data are marginal, but GLAST will be needed for a major increase in sensitivity and energy reach. Figure 6 shows one of the ways that GLAST will help - the smaller error bars on the theoretical curves show two models' predictions folded through the GLAST sensitivity for a one-year sky survey. GLAST will easily distinguish spectra such as these and might detect a second component at higher energies. A second strength of GLAST will be in its much higher sensitivity than previous instruments. Figure 7 shows one

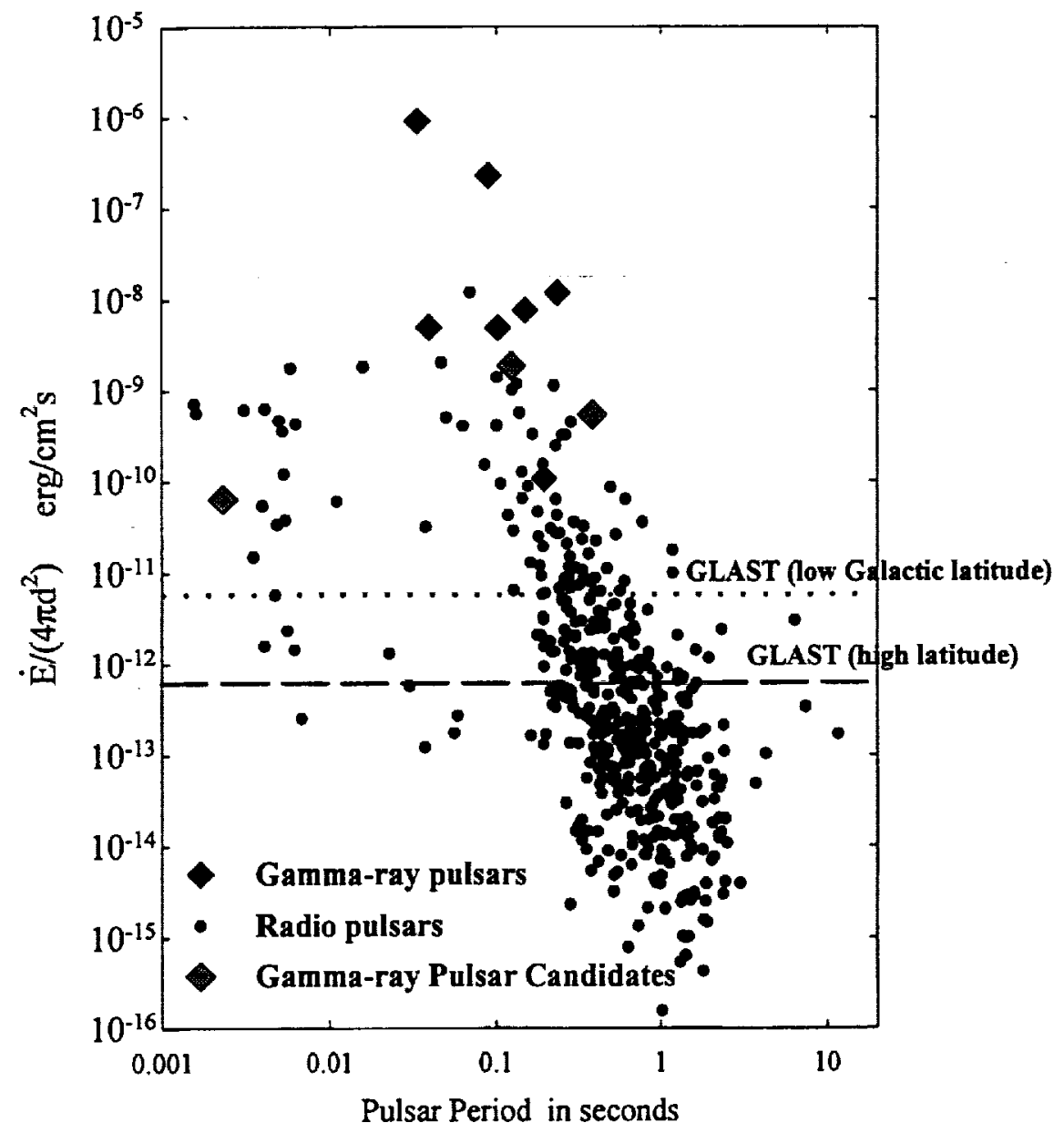

FIGURE 7. Gamma-ray pulsar observability, as measured by the spin-down energy seen at earth. 
of the classic measures of pulsar observability: the pulsar spin-down luminosity divided by $4 \pi$ times the square of the distance, the total available pulsar energy output at Earth. The 10 gamma-ray pulsars and candidates are shown as the large diamonds. Six of the seven pulsars with the highest value of this parameter are gamma-ray pulsars. Below these, only a handful of pulsars are visible in gamma rays. The GLAST sensitivity will push the lower limit down substantially farther. Two sensitivity limits are shown for GLAST - one for low-Galactic-latitude sources and one for those at high latitudes, because the high diffuse emission along the Galactic plane reduces the sensitivity for point source detection. The phase space that GLAST opens up is substantial.

How many pulsars will GLAST see? To some extent that number depends on which model best describes the emission and how the pulsars are distributed on the sky. One empirical estimate can be made by constructing a $\log N$-logS curve from the known pulsars. Figure 8 uses all nine of the high-energy gamma-ray pulsars to construct this function. The simple linear fit suggests that GLAST might expect to detect between 30 and 100 gamma-ray pulsars. The range of sensitivity is dependent

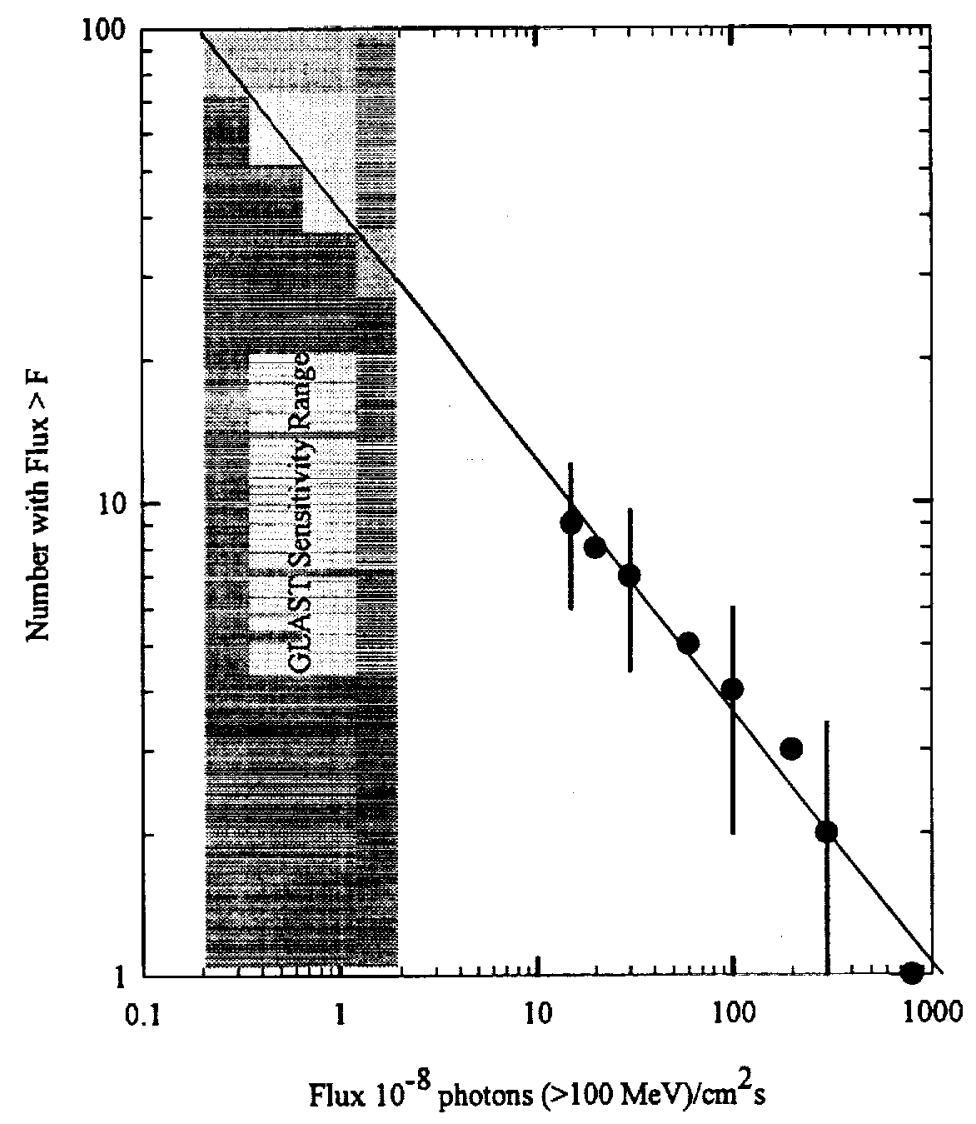

FIGURE 8. Cumulative number of gamma-ray pulsars seen by EGRET as a function of observed flux. Error bars are statistical and are shown on alternating data points for clarity. 
TABLE 1. Predicted gamma-ray fluxes (units of $10^{-8} \mathrm{ph}$ [E> $100 \mathrm{MeV}$ ] $\left.\mathrm{cm}^{-2} \mathrm{~s}^{-1}\right)$

\begin{tabular}{lrrrr}
\hline Pulsar J & $\begin{array}{r}\text { Zhang \& Harding } \\
{[29]}\end{array}$ & $\begin{array}{r}\text { Rudak \&Dyks } \\
{[30]}\end{array}$ & $\begin{array}{r}\text { Romani } \\
{[26]}\end{array}$ & $\begin{array}{r}\text { Cheng \&Zhang } \\
{[31]}\end{array}$ \\
\hline $1932+1059$ & $<580^{\mathrm{a}}$ & 90 & $\sim 0^{\mathrm{b}}$ & $<16$ \\
$0953+0755$ & $<500^{a}$ & 90 & $\sim 0^{b}$ & $<16$ \\
$0437-4715$ & $<230^{a}$ & $<2$ & $\sim 0$ & $<16$ \\
$2043+2740$ & 50 & 30 & $\sim 0^{b}$ & 50 \\
$1826-1334$ & 20 & 20 & $\leq 1$ & $<16$ \\
$1803-2137$ & 20 & 20 & 30 & $<16$ \\
$0742-2822$ & 20 & 20 & $\leq 7$ & 25 \\
$0117+5914$ & 20 & 20 & $\leq 35$ & 20 \\
$1801-2451$ & 15 & 10 & 23 & $<16$ \\
$1908+0734$ & 50 & 10 & $\sim 0$ & $<16$ \\
$1730-3350$ & 10 & 10 & $\leq 20$ & $<16$ \\
$0538+2817$ & 15 & 10 & $\leq 24$ & - \\
$0358+5413$ & 10 & 8 & $\leq 9$ & 20 \\
$1453-6151$ & 10 & $<2$ & $\sim 0^{b}$ & 16 \\
$2337+6151$ & 8 & $<2$ & $\leq 11$ & 20 \\
$1824-2452$ & 16 & $<2$ & $\leq 9$ & 20 \\
$1637-4553$ & 4 & $<2$ & $\leq 7$ & $<16$ \\
\hline a Calculated gamma-ray luminosity is a large fraction of the spin-down lumi- \\
nosity, hence an upper limit & & & $<16$ \\
b Observed gamma radiation expected to be small due to beaming &
\end{tabular}

on where the pulsars lie with respect to the Galactic plane. In some respects this figure is pessimistic, possibly due to the small sample. A disk population should increase linearly with decreasing sensitivity, but the fitted line goes as the -0.5 power of the sensitivity. A linear function would raise the number of pulsars to more than 100 .

Just as interesting as the number of pulsars to be seen by GLAST is which particular pulsars will be seen. Such observations are probably the best discriminator among models. Table 1 is a compilation with help from the theorists involved, showing the expected flux for various radio pulsars that have not yet been seen in gamma rays. For many of these, different models make predictions that differ by more than an order of magnitude. In these units, the GLAST sensitivity is 2 for pulsars near the plane and 0.2 for high-latitude pulsars; therefore GLAST will certainly provide solid observational tests of these and other gamma-ray pulsar models.

The third, and perhaps most important, capability of GLAST for pulsars will be its ability to find unknown pulse periods, in order to look for more Geminga-like pulsars. Various analyses have shown that only the brightest pulsars could be found in the EGRET data without independent information [32-34]. For all of the other unidentified EGRET sources, the photons are just too far apart in time to derive unambiguous pulse periods. With GLAST, periodicity searches will be feasible for 
most, if not all, the unidentified EGRET sources [35]. The potential is to find a whole new population of rotation-powered pulsars, much as $\mathrm{x}$-ray astronomy has started to do in the past few years. This could be an important new window onto the physics of the extreme conditions around these spinning neutron stars.

\section{SUMMARY}

- Gamma-ray pulsars remain a valuable probe of particle acceleration and interaction in the extreme conditions found near rotating neutron stars.

- At least 7 pulsars are seen in gamma rays (six of those at energies above 100 $\mathrm{MeV}$ ), with 3 additional good candidates.

- The changing shape of the light curves and energy spectra in the 1-20 GeV range make this band particularly interesting for future observations.

- GLAST, along with other satellite and ground-based gamma-ray telescopes, will make a major advance in gamma-ray pulsar studies.

\section{REFERENCES}

1. Thompson, D.J. , Adv. Space Res. 25, 659-668 (2000).

2. Becker, W., and Aschenbach, H. in Proc. NATO ASI, Vol. 450, Lives of the Neutron Stars, ed. M. A. Alpar, Ü. Kizilolglu, and J. van Paradijs, Dordrecht, Kluwer, 1995, pp. $47-51$.

3. Ulmer, M.P. et al., ApJ 432, 228-238 (1994).

4. Much, R. et al., in: Proceedings of the Fourth Compton Symposium, Williamsburg, $V A$, ed. C.D. Dermer, M.S. Strickman, and J.D. Kurfess, New York: AIP, AIP Conf. Proc. 410, 1997, 542-546.

5. Greiveldinger, C., Caucino, S., Massaglia, S., Ögelman, H., Trussoni, E., ApJ 454, 855-861 (1995).

6. Matz, S.M. et al., $A p J 434,288-291$ (1994).

7. Kuiper, L. et al., $A \mathcal{E} A, \mathbf{3 5 1}, 119-132$ (1999).

8. Ögelman, H., Finley, J.P., and Zimmerman, H.U., Nature, 361, 136-137 (1993).

9. Strickman, M. S., Harding, A. K., de Jager, O. C., ApJ 524, 373-378 (1999)

10. Kuiper, L. et al, in The Many Faces of Neutron Stars, ed R. Buccheri, J. van Paradijs, and M. A. Alpar, Dordrecht, Boston, Kluwer Academic Publishers, 1998., pp. 211227.

11. Kanbach, G., in The Many Faces of Neutron Stars, ed R. Buccheri, J. van Paradijs, and M. A. Alpar, Dordrecht, Boston, Kluwer Academic Publishers, 1998., pp.195211.

12. Thompson, D.J. et al., ApJ 465, 385-392 (1996).

13. Safi-Harb, S., Ögelman, H., and Finley, J.P., ApJ 439, $722-729$ (1995).

14. Kuiper, L., et al., $A \& A 337,421-432$ (1998).

15. Ramanamurthy, P.V. et al., $A p J 447$, L109-L112 (1995) 
16. Halpern, J.P. and Wang, F. Y.-H., ApJ 477, 905-915 (1997)

17. Thompson, D.J. et al., ApJ 516, 297-306 (1999)

18. Kaspi, V.M. et al., $A p J 528,445-453$ (2000).

19. Ramanamurthy, P.V. et al., ApJ 458, 755-760 (1996).

20. Kuiper, L. et al., $A B A$ 359, 615-626 (2000).

21. Taylor, J.H., Manchester, R.N. and Lyne, A.G., ApJS 88 , 529-568 (1993).

22. Arons, J., $A \mathscr{E} A S 120,49-60$ (1996).

23. Daugherty, J. K. and Harding, A. K., ApJ $458,278-292$ (1996).

24. Harding, A.K, private communication

25. Romani, R.W.,ApJ 470, 469-478 (1996)

26. Romani, R.W., private communication

27. Kanbach, G. et al.,AEA 289, 855-870 (1994).

28. Gehrels, N. and Michelson, P.,Astroparticle Phys. 11, 277-282 (1999).

29. Zhang, B. and Harding, A.K., A pJ 532, 1150-1171(2000).

30. Rudak, B., and Dyks, J.,MNRAS 295, 337-343 (1998).

31. Cheng, K.S. and Zhang, L., ApJ 498, 327-341 (1998).

32. Brazier, K.T.S. and Kanbach, G., $A \mathscr{E} A S 120,85-87$ (1996).

33. Mattox, J.R. et al., $A \& A S 120,95-98$ (1996)

34. Jones, B.B., PhD Thesis, Stanford University (1998)

35. Mattox, J.R., private communication. 